\title{
Strict versus liberal target range for perioperative glucose in patients undergoing coronary artery bypass grafting: A prospective randomized controlled trial
}

\author{
Shalin P. Desai, MD, Linda L. Henry, PhD, Sari D. Holmes, PhD, Sharon L. Hunt, MBA, \\ Chidima T. Martin, BS, CCRP, Shrinivas Hebsur, MD, and Niv Ad, MD
}

Objective: The purpose of this study was to test the hypothesis that a liberal blood glucose strategy (121-180
$\mathrm{mg} / \mathrm{dL})$ is not inferior to a strict blood glucose strategy $(90-120 \mathrm{mg} / \mathrm{dL})$ for outcomes in patients after first-time
isolated coronary artery bypass grafting and is superior for glucose control and target blood glucose management.

\begin{abstract}
Methods: A total of 189 patients undergoing coronary artery bypass grafting were investigated in this prospective randomized study to compare 2 glucose control strategies on patient perioperative outcomes. Three methods of analyses (intention to treat, completer, and per protocol) were conducted. Observed power was robust $(>80 \%)$ for significant results.
\end{abstract}

Results: The groups were similar on preoperative hemoglobin $\mathrm{A}_{1 \mathrm{c}}$ and number of diabetic patients. The liberal group was found to be noninferior to the strict group for perioperative complications and superior on glucose control and target range management. The liberal group had significantly fewer patients with hypoglycemic events ( $<60 \mathrm{mg} / \mathrm{dL} ; P<.001)$, but severe hypoglycemic events $(<40 \mathrm{mg} / \mathrm{dL})$ were rare and no group differences were found $(P=.23)$. These results were found with all 3 methods of analysis except for blood glucose variability, maximum blood glucose, and perioperative atrial fibrillation.

Conclusions: This study demonstrated that maintenance of blood glucose in a liberal range after coronary artery bypass grafting led to similar outcomes compared with a strict target range and was superior in glucose control and target range management. On the basis of the results of this study, a target blood glucose range of 121 to 180 $\mathrm{mg} / \mathrm{dL}$ is recommended for patients after coronary artery bypass grafting as advocated by the Society of Thoracic Surgeons. (J Thorac Cardiovasc Surg 2012;143:318-25)

Earn CME credits at

http://cme.ctsnetjournals.org

In recent years, data have shown that uncontrolled hyperglycemia is associated with poor clinical outcomes after surgical interventions. ${ }^{1-7}$ Likewise, control of glucose can improve outcomes in critically ill patients. Since the landmark study by Van den Berghe and colleagues ${ }^{2}$ in 2001, there have been guidelines for strict glycemic control in the intensive care unit (ICU). Over the past several years, the debate has been which patient population (surgical or medical) will benefit most from strict glycemic control and how strict a protocol is required..$^{1-7}$

From the Inova Heart and Vascular Institute, Falls Church, Va.

Disclosures: Authors have nothing to disclose with regard to commercial support.

Read at the 91st Annual Meeting of The American Association for Thoracic Surgery, Philadelphia, Pennsylvania, May 7-11, 2011.

Received for publication May 9, 2011; revisions received Oct 6, 2011; accepted for publication Oct 25, 2011; available ahead of print Dec 5, 2011.

Address for reprints: Niv Ad, MD, Inova Heart and Vascular Institute, 3300 Gallows Road, Suite \#3100, Falls Church, VA 22042 (E-mail: Niv.Ad@inova.org).

$0022-5223 / \$ 36.00$

Copyright (c) 2012 by The American Association for Thoracic Surgery

doi:10.1016/j.jtcvs.2011.10.070
Hyperglycemia is commonly encountered after cardiac surgery, whether a patient has a history of diabetes or not. ${ }^{1}$ Hyperglycemia has been associated with increased perioperative morbidity and mortality; several studies have demonstrated that glycemic control using insulin protocols improves operative mortality, lowers operative morbidity, and improves long-term survival. ${ }^{2-4}$

Although the consensus is that hyperglycemia should be managed after surgery, the optimal target serum glucose level that minimizes both perioperative complications and hypoglycemic events has yet to be established. In 2004, Lazar and colleagues ${ }^{6}$ demonstrated that maintaining the serum glucose between 120 and $180 \mathrm{mg} / \mathrm{dL}$ in diabetic patients undergoing cardiac surgery is safer and that a stricter regimen did not contribute to improved outcome. However, their study dealt only with diabetic patients, who comprise less than $50 \%$ of the operated population in the majority of centers. The Society of Thoracic Surgeons (STS) recommends that insulin infusions be titrated to maintain serum glucose at less than $180 \mathrm{mg} / \mathrm{dL}$ for the duration of the perioperative ICU stay. ${ }^{2,4}$ The recently completed Normoglycemia in Intensive Care Evaluation-Survival Using Glucose Algorithm Regulation (NICE-SUGAR) trial found that intensive glucose control (81-108 $\mathrm{mg} / \mathrm{dL}$ ) actually increased 90-day mortality compared with a more liberal glucose 


\section{Abbreviations and Acronyms \\ $\mathrm{BG} \quad=$ blood glucose \\ CABG $=$ coronary artery bypass grafting \\ ICU $\quad=$ intensive care unit \\ NICE-SUGAR $=$ Normoglycemia in Intensive \\ Care Evaluation-Survival \\ Using Glucose Algorithm \\ Regulation \\ STS $\quad=$ Society of Thoracic Surgeons}

target $(<180 \mathrm{mg} / \mathrm{dL})$ in patients in the ICU. ${ }^{7}$ However, this study was not focused solely on patents undergoing cardiac surgery and was mainly comprised of nonoperative patients, often with sepsis and multiorgan dysfunction.

Lazar and colleagues ${ }^{8}$ recently completed a prospective, randomized trial in diabetic patients undergoing coronary artery bypass grafting $(\mathrm{CABG})$ to determine whether tight glycemic control (90-120 mg/dL) would result in more optimal clinical outcomes than a more moderate glycemic control $(120-180 \mathrm{mg} / \mathrm{dL})$. They found that tight glycemic control increased the incidence of hypoglycemic events, but did not result in any significant improvement in clinical outcomes that was achieved with the more moderate control; however, this study also dealt only with diabetic patients. For the past several years, our patient management has dictated strict glycemic control with blood glucose (BG) levels maintained in the range of 90 to less than $120 \mathrm{mg} / \mathrm{dL}$ for all patients undergoing cardiac surgery. Our study aimed to compare the current practice of strict control with a liberal strategy: BG maintained at less than $180 \mathrm{mg} / \mathrm{dL}(121-180 \mathrm{mg} / \mathrm{dL})$.

\section{MATERIALS AND METHODS}

A randomized controlled study was performed to test the hypothesis that a liberal strategy of serum glucose control (BG 121-180 mg/dL) is not inferior to a strict strategy (BG $90-120 \mathrm{mg} / \mathrm{dL}$ ) in patients after first-time isolated $\mathrm{CABG}$ for complications and outcomes and is superior for glucose control and target BG management.

This study was a prospective, randomized controlled single-center clinical trial that examined adult patients undergoing first-time isolated CABG in whom hyperglycemia developed, thus requiring insulin therapy for treatment. All patients, regardless of a history of diabetes, were eligible for inclusion in this trial if they had 3 or more blood glucose readings of more than $150 \mathrm{mg} / \mathrm{dL}$ or $1 \mathrm{BG}$ reading more than $200 \mathrm{mg} / \mathrm{dL}$ (considered our standard of care). The study was approved by the Human Research Protection Program at Inova Heart and Vascular Institute (institutional review board number 09.111), and informed consent was obtained from all patients. This study was also registered on ClinicalTrials.gov.

\section{INCLUSION CRITERIA}

1. All diabetic patients who underwent first-time, isolated, nonemergency CABG.

2. Nondiabetic patients who underwent first-time, isolated, nonemergency CABG who were found to have had 3 consecutive $\mathrm{BG}$ readings greater than $150 \mathrm{mg} / \mathrm{dL}$ or any $1 \mathrm{BG}$ reading greater than $200 \mathrm{mg} / \mathrm{dL}$ perioperatively, which is aligned with the current STS guidelines.

3. Patients who were started on an insulin infusion while in the operating room.

\section{EXCLUSION CRITERIA}

1. Patients who underwent open surgery other than isolated CABG.

2. Patients who were found not to require an insulin infusion post-CABG.

3. Patients who underwent a concomitant procedure in addition to $\mathrm{CABG}$ (eg, CABG + valve repair).

\section{STUDY TREATMENT AND INTERVENTION}

Once enrolled, patients underwent a series of preoperative blood tests, anthropomorphic measurements, and assessment scales and inventories as noted in Figure 1. Questionnaires assessing depression (Patient Health Questionnaire 9), health-related quality of life (Short Form12), diet, and physical activity (Duke Activity Score) were filled out by patients preoperatively, although these results will not be included in the present analyses.

Intraoperative glucose measures and interventions were under the purview of the anesthesiologist, whose goal was to maintain a BG level between 100 and $180 \mathrm{mg} / \mathrm{dL}$. Maintenance of BG levels according to their randomized arm was started in the ICU using the programmed Glucommander (Gluco Tec, Greenville, SC) to adjust the BG level to patients' assigned range. The nursing staff was not blinded to treatment group allocation. Hourly BG monitoring was performed with blood obtained from a patient's arterial line and analyzed by point of care testing through Glucose Accu-Chek Advantage with the AccuData GTS/ GTS manufactured by Roche (Basel, Switzerland). BG levels less than $40 \mathrm{mg} / \mathrm{dL}$ or greater than $500 \mathrm{mg} / \mathrm{dL}$ were sent to the laboratory for further analysis; however, treatment was initiated for low BG if indicated. Patients were maintained on the electronic-based protocol of intravenous insulin for a minimum of 72 hours perioperatively.

\section{GLUCOMMANDER}

The Glucommander is a Food and Drug Administrationapproved computer software system for controlling BG designed to assist clinicians in obtaining and then maintaining glucose control by calculating the insulin dose required to achieve the target range in response to measurement of BG at the patient's bedside, but does not administer the insulin. It is the current method of glycemic control for patients post-CABG at our institution. We previously validated ${ }^{9}$ increased efficiency and efficacy of Glucommander data.

The Glucommander uses the variables of patients' height, weight, $\mathrm{HbA}_{1 \mathrm{C}}$, and current and cumulative $\mathrm{BG}$ values to 


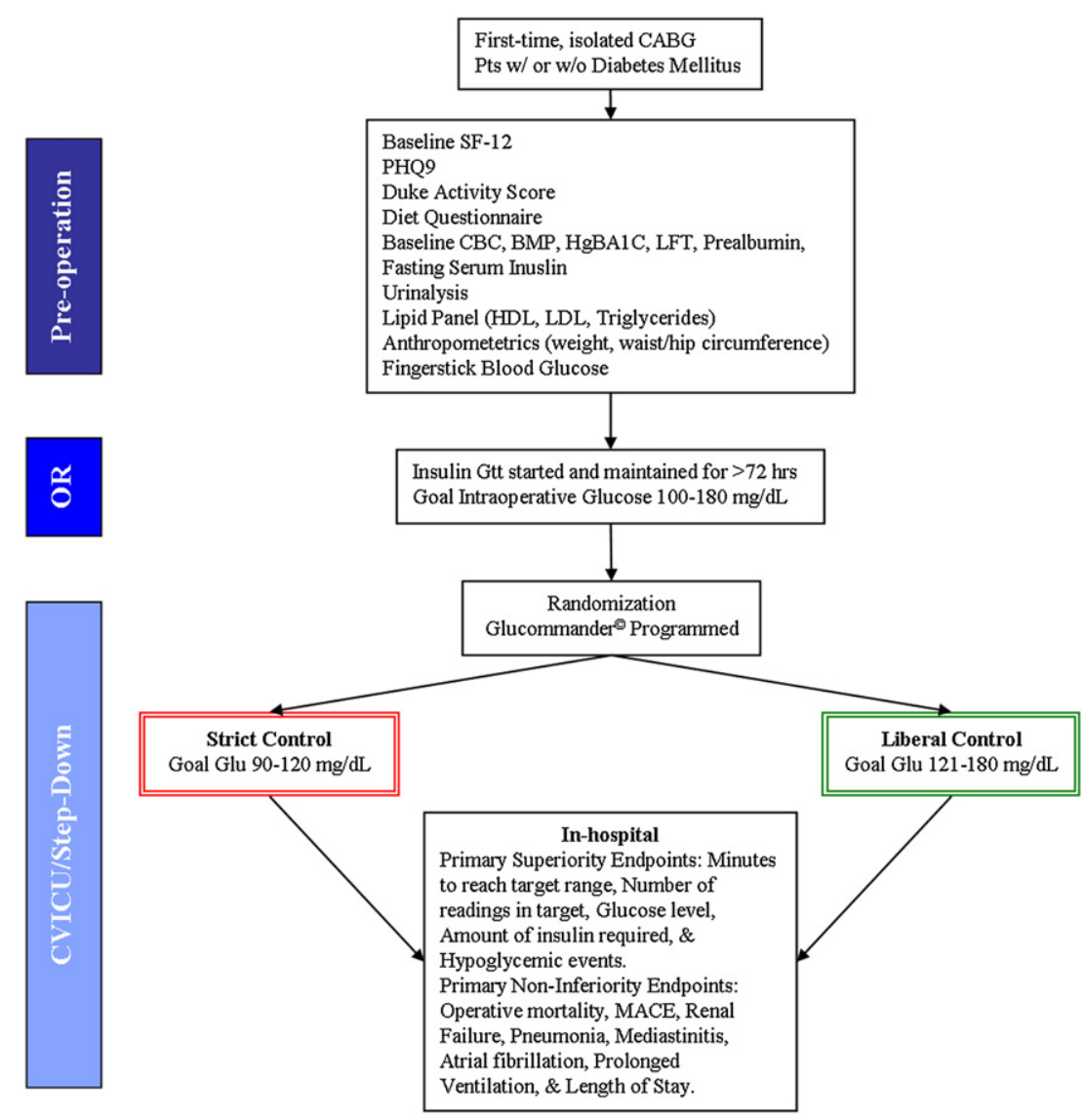

FIGURE 1. Trial design. CVICU, Cardiovascular intensive care unit; $O R$, odds ratio; $C A B G$, coronary artery bypass grafting; $P H Q 9$, Patient Health Questionnaire 9; $C B C$, complete blood count; $B M P$, basic metabolic panel; $H g B A 1 C$, hemoglobin $\mathrm{A}_{1 \mathrm{C}} ; L F T$, liver function test; $H D L$, high-density lipoprotein; $L D L$, low-density lipoprotein; Gtt, glucose tolerance test; Glu, glucose; MACE, major adverse cardiac event.

determine the algorithm-driven recommended dose of insulin or glucose to maintain individual patient BG levels in a predetermined range. The bedside nurses were responsible for carrying out any indicated intervention to include insulin infusion adjustment or glucose administration and further BG monitoring.

\section{RANDOMIZATION}

A block randomization process was used to randomly assign patients into the 2 groups (strict or liberal) with blocks of 10 patients. If the randomization scheme determined the patient was to be in the liberal arm, then an order was written in the chart to change the Glucommander parameters for a target glucose range of 121 to $180 \mathrm{mg} / \mathrm{dL}$. If the randomization scheme determined the patient was to be in the strict arm, then the patient continued on the standard CABG postoperative orders, mandating strict glycemic control with a target glucose range of 90 to $120 \mathrm{mg} / \mathrm{dL}$.

\section{END POINTS AND OUTCOMES MEASUREMENT}

The primary end points were 2-fold: superiority hypothesized for glucose control and target management and non- inferiority hypothesized for complications and outcomes. Superiority end points included time to target glucose range, amount of insulin given, number of readings in target range, and number of patients with hypoglycemic events $(\mathrm{BG}<60 \mathrm{mg} / \mathrm{dL}$ and $\mathrm{BG}<40 \mathrm{mg} / \mathrm{dL}$ ). Noninferiority end points included perioperative renal failure, deep sternal wound infection, pneumonia, length of stay, atrial fibrillation, and operative mortality (death within 30 days).

\section{DATA ANALYSIS}

The data for this article were managed using SAS/STAT software, version 9.1 of the SAS System (SAS Institute Inc, Cary, NC). Statistical analysis was performed with SPSS version 17.0 (SPSS Inc, Chicago, Ill). The groups were compared on categorical variables with the chi-square test or 2-sided Fisher exact test, as appropriate. Student $t$ test compared continuous variables. All outcomes initially were evaluated by intention-to-treat analyses. However, because of crossover between the 2 treatment arms, completer analyses (actual treatment received) and per protocol analyses (only those that received the treatment they were randomized to) were examined as a secondary step. A priori 


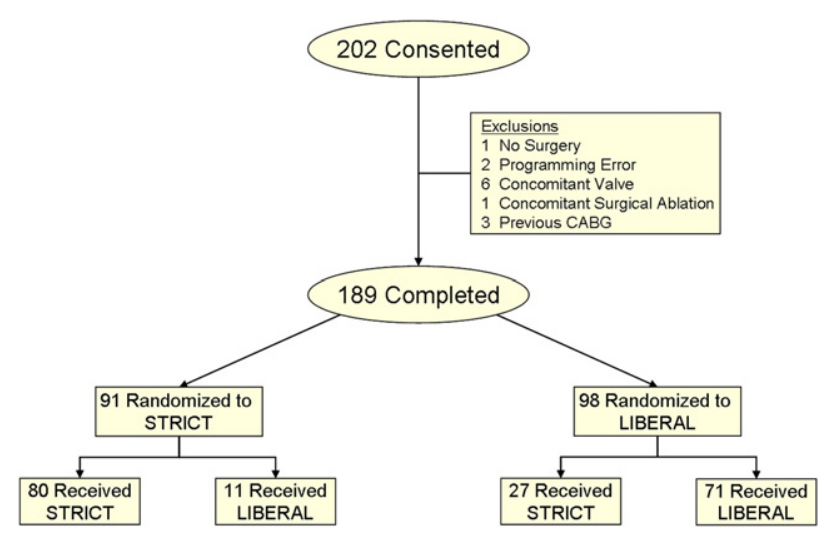

FIGURE 2. Flow of participants. $C A B G$, Coronary artery bypass grafting.

power analyses revealed $\mathrm{N}=180$ was sufficient for medium effect sizes with alpha set at 0.05 and power set at $80 \%$. Observed power was found to be robust $(>80 \%)$ for all analyses except those where no significance was found, given the smaller observed effect sizes for those comparisons.

We conducted noninferiority analyses to examine the effect of target range on clinical outcomes and complications. Noninferiority was declared if the effect difference and entire $95 \%$ confidence interval for the event rate in the liberal group did not exceed a relative margin of $10 \%$ $(-0.10$ to 0.10$)$ from the event rate in the strict group.

\section{RESULTS \\ Patient Characteristics}

This study randomized 189 patients to the strict or liberal target range (Figure 2). All results are reported using the intention-to-treat model, unless otherwise noted. The mean age of the patients was $62.7 \pm 9.8$ years, and $16 \%$ of the sample was female. There were no differences between the groups on baseline characteristics, including all markers from the preoperative blood panel and preoperative cardioprotective medications (Table 1). Diabetic patients were evenly distributed between the groups.

\section{Superiority of Glucose Control and Target Range Management}

Patients in the liberal group were found to reach their target range in less than half the time as the strict group (83.5 \pm 134.6 vs $173.3 \pm 195.8$ minutes; $t=3.65, P<.001$ ). The liberal group had more readings within the target range compared with the strict group ( $27.0 \pm 5.5$ vs $23.6 \pm 5.3$; $t=-4.31, P<.001)$, significantly fewer readings above target range ( $7.7 \pm 6.3$ vs $13.1 \pm 6.1 ; t=6.05, P<.001)$, and reduced variability (standard deviation) across the 72 hours ( $26.7 \pm 9.5$ vs $29.8 \pm 10.8 ; t=2.08, P=.04$ ). There was no difference between the liberal and strict groups on maximum BG $(218.3 \pm 46.8$ vs $213.3 \pm 48.2 ; t=-0.72$, $P=.47)$. The minimum BG level was higher in the liberal
TABLE 1. Patient characteristics (intention-to-treat; no significant group differences)

\begin{tabular}{lccc}
\hline & $\begin{array}{c}\text { Strict } \\
\mathbf{n = 9 1}\end{array}$ & $\begin{array}{c}\text { Liberal } \\
\mathbf{n = 9 8}\end{array}$ & $\begin{array}{c}\boldsymbol{P} \\
\text { value }\end{array}$ \\
\hline Age, y & $62.5 \pm 10.2$ & $62.8 \pm 9.5$ & .87 \\
Female & $10(11)$ & $20(20)$ & .11 \\
Diabetes & $37(41)$ & $44(45)$ & .56 \\
Hemoglobin $\mathrm{A}_{1 \mathrm{c}}$ & $6.64 \pm 1.52$ & $6.48 \pm 1.28$ & .44 \\
Hypertension & $75(82)$ & $84(86)$ & .56 \\
Congestive heart failure & $12(13)$ & $9(9)$ & .49 \\
Ejection fraction & $57 \pm 8$ & $56 \pm 9$ & .40 \\
Aspirin & $84(92)$ & $92(94)$ & .78 \\
Beta-blockers & $77(85)$ & $91(93)$ & .10 \\
ACE/ARB inhibitors & $46(51)$ & $46(47)$ & .62 \\
Lipid-lowering medications & $84(92)$ & $92(94)$ & .78 \\
STS risk percentage & $1.4 \pm 2.2$ & $1.1 \pm 2.1$ & .36 \\
euroSCORE (additive) & $3.97 \pm 2.98$ & $3.67 \pm 2.65$ & .47 \\
\hline ACE, Ang
\end{tabular}

$A C E$, Angiotensin-converting enzyme; $A R B$, angiotensin receptor blocker; euroSCORE, European System for Cardiac Operative Risk Evaluation.

group $(84.4 \pm 17.8$ vs $66.6 \pm 13.9 \mathrm{mg} / \mathrm{dL} ; t=-7.64$, $P<.001)$. The liberal group had fewer patients with at least 1 hypoglycemic event $(\mathrm{BG}<60 \mathrm{mg} / \mathrm{dL})$ than the strict group $(11[11 \%]$ vs 30 [33\%], $P<.001)$. However, there was not a difference in the number of patients who experienced severe hypoglycemia (BG $<40 \mathrm{mg} / \mathrm{dL}$ ) between the groups ( 0 vs 2 [2.2\%], $P=.23$ ), but the occurrence of these events was rare. The number of readings did not differ between the groups $(t=1.01, P=.31)$, a factor controlled by the Glucommander system. Last, the liberal group required fewer total units of insulin compared with the strict group $(166.0 \pm 156.7$ vs $252.3 \pm 193.4 ; t=3.36$, $P<.001)$.

\section{Noninferiority of Perioperative Complications and Outcomes}

Event rates were small for perioperative complications and clinical outcomes. There were no incidences of perioperative myocardial infarction, permanent stroke, or leg

TABLE 2. Results of the noninferiority analyses for clinical outcomes (intention-to-treat)

\begin{tabular}{lcccc}
\hline & $\begin{array}{c}\text { Strict } \\
\mathbf{n}=\mathbf{9 1}\end{array}$ & $\begin{array}{c}\text { Liberal } \\
\mathbf{n = 9 8}\end{array}$ & $\begin{array}{c}\text { Treatment } \\
\text { difference }\end{array}$ & $\mathbf{9 5 \%} \mathbf{C I}$ \\
\hline Renal failure & $2.2 \%$ & 0 & -0.022 & -0.05 to 0.008 \\
Atrial fibrillation & $8.0 \%$ & $10.2 \%$ & 0.022 & -0.06 to 0.10 \\
Pneumonia & $2.2 \%$ & 0 & -0.022 & -0.05 to 0.008 \\
$\begin{array}{l}\text { Deep sternal wound } \\
\quad 1.1 \%\end{array}$ & 0 & -0.011 & -0.03 to 0.01 \\
$\quad$ infection & & & & \\
Prolonged ventilation & $3.3 \%$ & 0 & -0.033 & -0.07 to 0.004 \\
$\quad(>24$ h) & & & & \\
MACE & $2.2 \%$ & $2.0 \%$ & -0.002 & -0.04 to 0.04 \\
LOS $>10$ d & $3.3 \%$ & $2.0 \%$ & -0.013 & -0.06 to 0.03 \\
Operative mortality & $1.1 \%$ & $1.0 \%$ & -0.001 & -0.03 to 0.03 \\
\hline
\end{tabular}

$C I$, Confidence interval; $M A C E$, major adverse cardiac event; $L O S$, length of stay. 
A
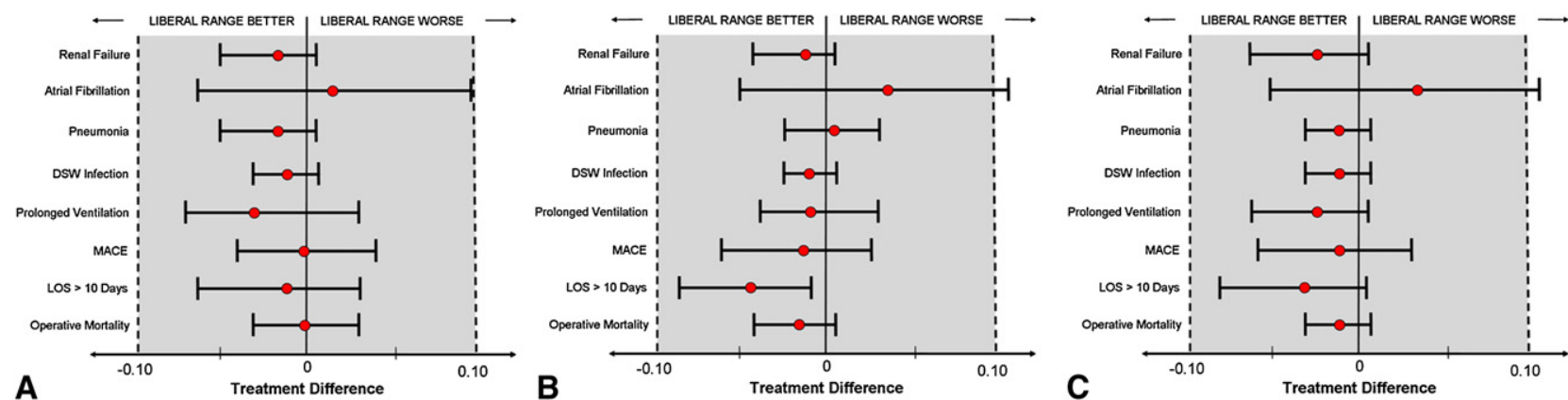

FIGURE 3. Treatment difference and $95 \%$ confidence interval for noninferiority of perioperative complications and outcomes: intention-to-treat analyses (A), completer analyses (B), and per protocol analyses (C). DSW, Deep sternal wound infection; MACE, major adverse cardiac events; $L O S$, length of stay.

infections for both groups. The liberal group was found to be noninferior to the strict group on all outcome variables (Table 2 and Figure 3, A).

\section{Completer Analyses (as Treated)}

As shown in Figure 2, 11 patients randomized to the strict group received the liberal target range and 27 patients randomized to the liberal group received the strict range due to clinical practice factors beyond the control of the study, including caregiver personnel changing or failing to change the preset target range on the Glucommander device. To ensure bias was not introduced in these results, completer analyses were conducted to examine the target range groups based on what the patient actually received (liberal $=82$, strict $=107)$. These analyses revealed that all superiority and noninferiority analysis results were similar to that of the intention-to-treat analyses. The only exceptions were found for maximum BG, BG variability, and perioperative atrial fibrillation. In the completer analyses, the liberal group was not different on variability $(27.4 \pm 11.2$ vs $28.8 \pm 9.5 ; t=0.93, P=.35)$ and had significantly higher maximum BG compared with the strict group $(229.0 \pm 49.6$ vs $205.9 \pm 43.3 ; t=-3.41, P<.001$ ), whereas this effect was attenuated in the intention-to-treat analysis and no significant difference was found $(t=-0.72, P=.47)$. Although the treatment difference for perioperative atrial fibrillation (0.035) was within the noninferiority margin, the entire $95 \%$ confidence interval was not $(-0.05$ to 0.12 ; Figure 3 , $B$ ) and therefore did not meet our criteria for noninferiority.

\section{Per Protocol Analyses}

A third method of analysis was conducted to examine those patients whose actual treatment arm matched their randomized group (liberal $=71$, strict $=80$ ). Again, superiority and noninferiority analysis results were similar to that of the intention-to-treat analyses with the exceptions of maximum BG, BG variability, and perioperative atrial fibrillation. In the per protocol analyses, the liberal group was not different on variability $(26.4 \pm 9.8$ vs $29.3 \pm 9.7$; $t=1.78, P=.08)$ and had significantly higher maximum BG compared with the strict group $(225.9 \pm 47.7$ vs
$208.5 \pm 44.9 ; t=-2.32, P=.02)$. The treatment difference for perioperative atrial fibrillation $(0.036)$ was within the noninferiority margin, but not the $95 \%$ confidence interval $(-0.05$ to 0.12 ; Figure $3, C)$.

\section{DISCUSSION}

Regardless of the method of analysis, the liberal group was significantly superior to the strict group on the outcomes of time to target $\mathrm{BG}$ range, the number of patients with hypoglycemic events ( $\mathrm{BG}<60 \mathrm{mg} / \mathrm{dL}$ ), and the number of readings that $\mathrm{BG}$ levels were within their respective target ranges. The more liberal group (BG target range of $121-180 \mathrm{mg} / \mathrm{dL}$ ) had a quicker time to target BG range after admission to the cardiovascular ICU, was maintained within its specified target range, had fewer patients with hypoglycemic events, and was not inferior on perioperative complications. The liberal group was also noninferior to the strict group in the perioperative outcomes of prolonged ventilation, deep sternal wound infection, pneumonia, perioperative renal failure, and operative mortality. On the basis of these results, it seems reasonable to set the target BG range to between 121 and $180 \mathrm{mg} / \mathrm{dL}$ for this group of patients undergoing first-time isolated CABG.

These findings are consistent with the recommendations of Lazar and colleagues ${ }^{5,6}$ and the STS guidelines that were developed from a series of clinical trials to include the work by Lazar and colleagues. Currently, the STS has recommended that patients undergoing cardiac surgery have their BG levels maintained at less than $180 \mathrm{mg} / \mathrm{dL}$ while in the ICU (level A) and that the most efficient way to achieve glucose control is with insulin infusions (level A). They further suggest that postprandial BG levels on the stepdown unit should also be maintained at less than $180 \mathrm{mg} / \mathrm{dL}$ (level B) using oral agents if previously prescribed or subcutaneous basal and bolus insulin therapy, such as glargine and lispro. ${ }^{5}$ All our study patients were maintained on insulin infusions for the first 72 hours if they had 3 sequential BG levels greater than $150 \mathrm{mg} / \mathrm{dL}$ immediately perioperatively. On the morning of the third day, patients were subsequently weaned from the insulin drip and started on prior prescribed medication or insulin 
subcutaneous injections if necessary. As noted, the patients in the liberal group (BG 121-180 mg/dL) fared better in glucose control requiring less insulin, were in target more often, and had a smoother transition off their insulin infusions than the patients in the strict control group (BG 90-120 mg/dL).

Our findings are also in line with the just released American College of Physicians guidelines for intensive insulin therapy in hospitalized patients. Although the guidelines are not specific to the cardiac surgical patient, they do address patients in the ICU (surgical and mobile ICU). The task force recommends against using intensive-insulin therapy, but rather using conventional glucose control mechanisms to achieve BG control. However, they also state that if intensive insulin therapy is used then the BG target level should be kept between 140 and $200 \mathrm{mg} / \mathrm{dL} .{ }^{10}$ The average BG level was $133 \pm 17$ (range 98-183) in the liberal group during the first 24 hours after surgery, $134 \pm 20$ (range 95-181) for the second 24 hours, and $124 \pm 18$ (range 90-166) for the third 24 hours, which is closely in line with these recommendations.

The NICE-SUGAR trial was recently completed, and the findings indicate that in this large, international randomized trial intensive glucose control increased mortality among adults in the ICU. The investigators concluded that a BG target of less than $180 \mathrm{mg} / \mathrm{dL}$ was an appropriate level to achieve. $^{7}$

Our study design was also similar to the NICE-SUGAR trial. In the NICE-SUGAR trial, 6104 patients were randomized to an intensive glucose control arm (target BG of $81-108 \mathrm{mg} / \mathrm{dL}$ ) or a conventional control arm (target BG of $<180 \mathrm{mg} / \mathrm{dL}$ ), and patients were enrolled from both medical and surgical ICUs. In our study, we randomized patients to an intensive glucose control arm, with a target range of 91 to $120 \mathrm{mg} / \mathrm{dL}$, or to a liberal control arm, with a target BG range of 121 to $180 \mathrm{mg} / \mathrm{dL}$. In the NICE-SUGAR trial, $6.8 \%$ of the patients in the intensive control group experienced severe hypoglycemia (BG level $\leq 40 \mathrm{mg} / \mathrm{dL}$ ), which was significantly different than the $0.5 \%$ of the patients in the conventional treatment group $(P<.001){ }^{7}$ Our findings were similar in that patients in the strict glucose control group did have significantly $(P<.001)$ more hypoglycemic episodes $(<60 \mathrm{mg} / \mathrm{dL}$; $33 \%)$ than the liberal group (11\%), although the groups were not significantly different in the number of patients with severe hypoglycemia $(<40 \mathrm{mg} / \mathrm{dL} ; 2.2 \%$ vs $0 \%$, $P=.23$ ). It is possible that there was not a significant difference between the 2 groups in severe hypoglycemia because of the use of the computer software program (Glucommander) for insulin management. The Glucommander instructed the bedside nurse on exactly what to administer according to the glucose level, so there was no delay in treatment. We also noted that most of the hypoglycemic events occurred late on the second day and into the third day around the time the patient had started to eat a regular diet causing the blood glucose to spike. The spike in the blood glucose caused the computer algorithm to adapt, but did not allow for an expected decrease in the postprandial BG level. This issue is currently being addressed as Glucommander updates its algorithm to allow for patients eating a regular diet. ${ }^{9}$

A rather striking finding of our study is the amount of time we found that patients actually were within their assigned target range, that is, the variability of $B G$ values. We found the strict group spent significantly less time within their target range than the more liberal group despite hourly blood draws and insulin adjustments. Using the intention-to-treat analysis, there was also a significant difference between the groups on standard deviation across the 72-hour time period. Several recent studies have actually questioned whether it is the variability of the BG values rather than the mean that influenced outcomes. ${ }^{11-18}$ Egi and Bellomo ${ }^{11}$ postulate that the difference in variability of BG control may be the reason why intensive insulin therapy has varied from beneficial to harmful, especially when comparing the Leuven ${ }^{19}$ (higher variability in the control arm) trial with the NICE-SUGAR ${ }^{7}$ (equivalent in both arms) trial and their respective outcomes and recommendations.

Another strength of this study was that all 3 methods of analyses maintained robust power. The a priori sample size of this study $(\mathrm{N}=200)$ was determined to be sufficient using a medium effect size, an alpha level of 0.05 , and $80 \%$ power. ${ }^{20}$ Observed power was found to be robust for significant results, but not robust for nonsignificant results as expected, given the much smaller observed effect sizes for those comparisons. The strong power of this study allowed us to detect significant results on almost all of the superiority analyses and noninferiority on the clinical outcomes. However, the fact that we were unable to find noninferiority on atrial fibrillation using the completer and per protocol analyses may be because this particular perioperative outcome has a small event rate at our facility. At Inova Heart and Vascular Institute, we aggressively pretreat all our patients undergoing cardiac surgery with amiodarone such that our institutional event rate $(10 \%-13 \%)$ is well below the national average as reported by the STS $(23 \%-24 \%)$ and was only $9 \%$ in this study. ${ }^{21}$ Therefore, more patients are needed to investigate this particular outcome before any conclusion may be drawn.

Our findings are in contradiction to those of Furnary and colleagues $^{3}$ and Furnary and $\mathrm{Wu},{ }^{4}$ who recommend tight glycemic control $(<120 \mathrm{mg} / \mathrm{dL})$ be obtained in all patients. ${ }^{3,4}$ We demonstrated in a prospective randomized controlled study that for patients undergoing first-time isolated CABG, a BG of less than $180 \mathrm{mg} / \mathrm{dL}$ is acceptable and not inferior to the less than $120 \mathrm{mg} / \mathrm{dL}$ in perioperative outcomes. In fact, the less than $180 \mathrm{mg} / \mathrm{dL}$ BG range was found to be superior when obtaining and maintaining the target range. We hypothesize that our different findings may be 
due to the in-depth detail we now have because of the computerization of our intensive insulin therapy. We are able to look beyond just the average BG values to have a better understanding of what may be the main factor affecting patient outcomes related to BG management.

\section{Study Limitations}

A possible limitation is that this was not a blinded study, which could introduce bias into the study. However, because a computer software program was used to determine the rate of the insulin infusion and any glucose administration, the caregiver was unable to indirectly influence a patient's BG levels.

Another possible limitation is that we did not draw markers of inflammation. Some studies have found that tight glycemic control affects these inflammatory markers and thus may affect long-term patency of the bypass grafts and reduce ischemic events. ${ }^{22-24}$

\section{CONCLUSIONS}

The findings of this study suggest that tight glucose control $(90-120 \mathrm{mg} / \mathrm{dL})$ in patients undergoing CABG may not be warranted in the short term. Therefore, we suggest that all patients who undergo CABG should have a more liberal BG target level of 120 to $180 \mathrm{mg} / \mathrm{dL}$ while hospitalized, which is line with the current STS guidelines. Further work is needed to determine the optimal BG level for long-term benefit and the optimal level for patients undergoing surgery for valvular heart disease.

\section{References}

1. Inzucchi SE. Management of hyperglycemia in the hospital setting. $N$ Engl J Med. 2006;335:1903-11.

2. Van den Berghe G, Wouters P, Weekers F, Verwaest C, Bruyninckx F, Schetz M, et al. Intensive insulin therapy in critically ill patients. $N$ Engl J Med. 2001;345: 125-39.

3. Furnary AP, Gao G, Grukenmeier GL, Wu Y, Zerr KJ, Bookin SO, et al. Continuous insulin infusion reduces mortality in patients with diabetes undergoing coronary artery bypass grafting. J Thorac Cardiovasc Surg. 2003;125: 1007-21.

4. Furnary AP, Wu TX. Clinical effects of hyperglycemia in the cardiac surgery population: the Portland Diabetic Project. Endocr Pract. 2006;12:22-6.

5. Lazar HL, McDonnell M, Chipkin S, Furnary AP, Furnary AP, Engelman RM, et al. Society of Thoracic Surgeons Blood Glucose Guideline Task Force. The Society of Thoracic Surgeons practice guideline series: blood glucose management during adult cardiac surgery. Ann Thorac Surg. 2009;87:663-9.

6. Lazar HL, Chipkin S, Fitzgerald C, Bao Y, Cabral H, Apstein C. Tight glycemic control in diabetic coronary artery bypass graft patients improves perioperative outcomes and decreases recurrent ischemic events. Circulation. 2004;109: 1497-502.

7. The NICE-SUGAR Study Investigators. Intensive versus conventional glucose control in critically ill patients. N Engl J Med. 2009;360:1283-97.

8. Lazar HL, McDonnell M, Chipkin S, Fitzgerald C, Bliss C, Cabral H. Effects of aggressive versus moderate glycemic control on clinical outcomes in diabetic coronary bypass graft patients. Ann Surg. 2011;254:458-64.

9. Halpin L, Henry L, Dunning E, Hunt S, Barnett S, White J, et al. Comparison of blood glucose management strategies to achieve control following cardiac surgery (computerized versus paper). AACN Adv Crit Care. 2010;21:146-51.

10. Qaseem A, Humphry L, Chou R, Snow V, Shekelle P. Use of intensive insulin therapy for management of glycemic control in hospitalized patients: a clinical practice guideline from the American College of Physicians. Ann Intern Med. 2011;154:260-8.

11. Egi M, Bellomo R. Reducing glycemic variability in intensive care unit patients: a new therapeutic target? J Diabetes Sci Technol. 2009;3:1302-8.

12. Egi M, Bellomo R, Stachowski E, French C, Hart G, Stow P, et al. Intensive insulin therapy in perioperative intensive care patients: a decision analysis. Am J Respir Care Med. 2006;173:407-13.

13. Egi M, Bellomo R, Stachowski E, French C, Hart G, Stow P. Circadian rhythm of blood glucose values in critically ill patients. Crit Care Med. 2007;35: 416-21.

14. Ali N, O’Brien J Jr, Dungan K, Phillips G, Marsh C, Lemeshow S, et al. Glucose variability and mortality in patients with sepsis. Crit Care Med. 2008;36: 2316-21.

15. Hirshberg E, Larsen G, Van Duker H. Alterations in glucose homeostasis in the pediatric intensive care unit: hyperglycemia and glucose variability are associated with increased mortality and morbidity. Pediatr Crit Care Med. 2008;9: 361-6.

16. Dossett L, Cao H, Mowery N, Dortch M, Morris J Jr, May A. Blood glucose variability is associated with mortality in the surgical intensive care unit. Am Surg. 2008;74:679-85.

17. Waeschle R, Moerer O, Hilgers R, Herrmann P, Neumann P, Quintel M. The impact of the severity of sepsis on the risk of hypoglycemia and glycaemic variability. Crit Care. 2008;12:R129.

18. Krinsley JS. Glycemic variability: a strong independent predictor of mortality in critically ill patients. Crit Care Med. 2008;36:3008-13.

19. Vanhorebeek I, Ingels C, Van den Berghe G. Intensive insulin therapy in high-risk cardiac surgery patients: evidence from the Leuven randomized study. Semin Thorac Cardiovasc Surg. 2006;18:309-16.

20. Cohen J. Statistical Power Analysis for the Behavioral Sciences. 2nd ed. Hillsdale, NJ: Lawrence Erlbaum Associates; 1988

21. Society of Thoracic Surgeons. STS Adult Surgery Database Report from the Duke Research Institute 2010. Available at: http://www.sts.org/sites/default/ files/documents/pdf/ndb2010/Report_OV_General_5-37.pdf. Accessed November 16, 2011.

22. Koskenkari J, Kaukoranta P, Rimpilainen J, Vainonpaa V, Ohtonen P, Surcel HM, et al. Anti inflammatory effect of high dose insulin treatment after urgent coronary revascularization surgery. Acta Anaesthesiol Scand. 2006;50:962-9.

23. Visser L, Zuurbrier C, Hoek F, Opmeer B, deJonge E, de Mol B, et al. Glucose, insulin and potassium applied as perioperative hyperinsulinaemic normoglycemic clamp: effects on inflammatory response during coronary artery surgery. Br J Anaesth. 2005;95:448-57.

24. Chaduri A, Janicke D, Wilson M, Tripathy D, Garg R, Bandyopabhyay A, et al. Anti-inflammatory and profibrinolytic effect of insulin in acute ST segment elevation myocardial infarction. Circulation. 2004;109:849-54.

\section{Discussion}

Dr Harold Lazar (Boston, Mass). I enjoyed your presentation and appreciate you sending me the manuscript well ahead of time. My only conflict is that I have received grant support from the Eli Lilly Company to study the effects of glycemic control during cardiac surgery.

Last month at the American Surgical Association, my colleagues and I presented a article in which we prospectively randomized 82 diabetic patients undergoing CABG to receive an aggressive glycemic group, 90 to 120 , or a moderate group, 120 to 180 . We found no difference in 30-day mortality, myocardial infarctions, neurologic complications, deep sternal wound infections, or incidence of atrial fibrillation between groups, which was similar to your results. The patients with more aggressive control had a higher incidence of hypoglycemic events, but this did not result in any neurologic complications. We also concluded that aggressive glycemic control increases the incidence of hypoglycemic events but does not result in any significant improvement in clinical outcomes that can be achieved with more moderate control. 
Although I am not surprised that moderate control is safe and effective, the question is, why does more aggressive glycemic control offer no further advantages and is potentially harmful? Your study and our own data may provide some potential mechanisms for answering these questions.

First, many patients undergoing CABG are already receiving cardioprotective agents. For example, in our own study, $100 \%$ of patients were already receiving aspirin, a statin, and a betablocker, and more than $50 \%$ of patients received an angiotensin-converting enzyme inhibitor. Thus, the added benefits of aggressive glucose lowering may not have as great an effect in reducing cardiovascular events as anticipated. So my first question is, what was the incidence of use of these agents between the groups in your own study?

Second, in our study, moderate glycemic control achieved an average serum glucose of 135 versus 103 for the aggressive group. Thus, even when aiming for more moderate control, tight glycemic control was still achieved. We found that using our paper algorithm, both the moderate and aggressive groups remained in their respective target ranges $100 \%$ of the time. Do you think that the computerized Glucommander system could have accounted for the wider variation in your patients receiving the more aggressive control? Less than $50 \%$ of patients in your study were diabetic. Could the larger incidence of nondiabetic patients have made tighter glycemic control more vulnerable to these hypoglycemic episodes?

Third, we have shown in our series that one of the benefits of glycemic control is that markers of inflammation, such as free fatty acids, are significantly reduced. In our recent study, aggressive control actually resulted in a significant decrease in free fatty acids compared with the more moderate control. This ultimately may result in long-term benefits, such as improved graft patency and reduced ischemic events. Did you measure any inflammatory markers and do you have any long-term follow-up for these patients as to the incidence of recurrent ischemic episodes and the need for re-revascularization? It may turn out that more aggressive control might still have some hidden long-term benefits.
Finally, as we have seen in our earlier studies, moderate glycemic control alone has resulted in a significant decrease in morbidity and mortality, which may be difficult to improve on with more aggressive control.

In summary, although I agree that the optimal range for glycemic control in cardiac surgery patients is 120 to 180 , we should all remember that although the exact value for achieving optimal perioperative glycemic control is still unknown and the subject of numerous studies and intense debate, the concept of perioperative glycemic control using continuous insulin infusions in our patients is well established and its importance is not debatable.

Dr Desai. In terms of whether we had data regarding cardiac medications that the patients were taking, we did collect medication data for all the patients who were enrolled in the study, but this was not analyzed. In regard to inflammatory markers, we did measure erythrocyte sedimentation rate and C-reactive protein levels in all patients who were enrolled in this study. I do not have the data here on the levels in the strict group versus the liberal group.

In terms of the computer algorithm versus the paper algorithm and the wide variation, I think having our study open to a lot of nondiabetic patients did contribute to the wide variability. These are patients who are not normally hyperglycemic, but because of the stress of surgery and the other factors involved, their glucose levels were severely elevated to the point that they are started on insulin infusions. We also noticed that once we got to approximately 48 hours after the surgery, the patients were starting to take an oral diet. Because of this, we also noticed large spikes in their glucose, which the computer algorithm does not currently into account. Deviations from target range was common because of this phenomenon and have been seen in other similar studies. It is excellent, Dr Lazar, that your study was able to maintain patients $100 \%$ in their target range. We believe further work needs to be done with the Glucommander computerized system in regard to adjusting the algorithm once the patients start taking an oral diet after their surgery to maintain their target range. 